\title{
Coix seed improves growth performance and productivity in post-weaning pigs by reducing gut $\mathrm{pH}$ and modulating gut microbiota
}

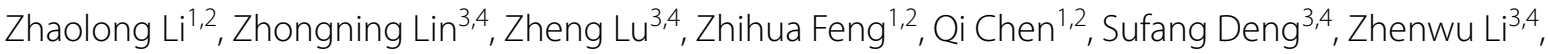 \\ Youquan Yan ${ }^{2,3}$ and Zhaoyang Ying ${ }^{3,4^{*}}$
}

\begin{abstract}
Coix seed has traditionally been used in traditional Chinese medicine to fortify the spleen and inhibit dampness, and has shown anticancer effects in humans. However, it is not known whether coix seed improves post-weaning growth performance and productivity, and the mechanism of interaction between coix seed and gut microbiota remains unknown. In this study, we established four groups: (i) control, (ii) antibiotic-fed, (iii) coix seed powder-fed, and (iv) coix seed extract-fed. The feeding experiment was conducted for 4 weeks. Coix seed extract significantly increased average weight gain and reduced the feed/meat ratio in weaned pigs, in addition to reducing the $\mathrm{pH}$ of their gastric juice. Further assays demonstrated that coix seed promotes an increase in the density and length of the gastrointestinal villi. Next, 16 s sequencing of gut microbiota showed that coix seed significantly increased the abundance of phylum Bacteroidetes and genus Lactobacillus $(p<0.05)$ and reduced the abundance of phylum Prevotella $(p<0.05)$ in the gut microbiota. In contrast, the abundance of phylum Bacteroidetes and genus Lactobacillus decreased in the control group and antibiotic group, whereas the abundance of phylum Prevotella increased. Our findings indicate that coix seed improves growth performance and productivity in post-weaning pigs by reducing the $\mathrm{pH}$ value of gastric juice, increasing the density and length of gastrointestinal villi, and modulating gut microbiota. Thus, coix seed has good potential for use as a feed supplement in swine production.
\end{abstract}

Keywords: Coix seed, Piglets, Gut microbiota, Villi, pH

\section{Introduction}

Weaning transition is a critical period in the growth of piglets (Wolter et al. 2002). During this period, the digestive and immune systems are in their developmental phases, and piglets experience a wide variety of stresses, such as an abrupt shift in diet from high-fat, low-carbohydrate breast milk to high-carbohydrate and low-fat feed, which can lead to poor health and growth performance (Thexton et al. 1998). Thus, antibiotics are widely used to treat bacterial infections and stress-induced

\footnotetext{
*Correspondence: zhaoyang_ying@163.com

${ }^{3}$ Agricultural Ecology Institute, Fujian Academy of Agricultural Sciences,

Pudang, Jin-an District, Fuzhou 350013, Fujian, China

Full list of author information is available at the end of the article
}

gastrointestinal dysfunction. Misuse and overuse of antibiotics increase antimicrobial resistance, which is a serious threat for human, animal, and environmental health (Stein and Dong 2006; Coffin et al. 2013; Gellin et al. 1989; Lee et al. 2000). This threat has been increasingly recognized, and several solutions have been proposed. These include reducing or limiting the use of antibiotics and strictly checking the excess of antibiotics used in animal production (Centner 2016; Spiro et al. 2011; Pettigrew 2012; Van Der Fels-Klerx et al. 2011); however, the threat still persists (Hamscher et al. 2003; Sapkota et al. 2006; Murphy et al. 2007; Ferguson et al. 2016). Herbal alternatives such as traditional Chinese medicine (TCM) are widely accepted as high-efficiency and low-toxicity "medicinal diets" that are capable of preventing certain 
side effects (Williamson et al. 2013; Hsiao and Liu 2010; Normile 2013; Fu et al. 2015). Moreover, the majority of TCM treatments also contain certain food components that provide benefits beyond basic nutrition (Bao 2017); therefore, some TCMs can be used in the animal industry as alternative supplements to modulate gut macrobiota and promote growth, as well as to reduce the threat posed by antibiotics to animal and human health (Gao et al. 2010; Zhang et al. 2008; Hui et al. 2011; Jin et al. 2008; Li et al. 2008; Chen et al. 2009). In addition, most TCMs are rich in polysaccharides, polyphenols, phytosterols, and lactams, which are increasingly recognized for their anti-cancer, anti-oxidant, and anti-inflammatory effects; endocrine regulation; blood pressure and fat reduction; and immune regulation (He and Dai 2011; Zhao et al. 2014; Zhou et al. 2017).

Coix seed belongs to the family Poaceae, and is widely distributed in China, Japan, Thailand, and Burma. Coix seed contains a high content of essential amino acids, lipids, proteins, polysaccharides, polyphenols, phytosterols, and lactams, and has favorable physiological and pharmacological effects, including those mentioned above. As such, it has long been used in TCM (Liu et al. 2010; Kaneda et al. 1992; Tokuda et al. 1990; Qu et al. 2016; Yu et al. 2008). Coix seed has also been used as a substitute for rice or wheat in animal feed because of its high starch, oil, and protein content (Yu et al. 2008; Weng 2013; Jin et al. 2011). However, the use of coix seed for anti-stress effects during the weaning transition process has not been reported. Only a handful of studies have shown that the beneficial effects of coix seed might be associated with gastrointestinal microorganisms (Liu et al. 2019).

In this study, we investigated the effects of coix seed on the weaning transition in piglets and its subsequent effects on gastrointestinal villi, gastrointestinal $\mathrm{pH}$, and the composition of gastrointestinal microbiota. Coix seed promoted higher average weight of piglets and a lower feed/meat ratio, which was associated with alteration in the structure of the gut microbiota and gut $\mathrm{pH}$. The gut microbiota is known to respond to fluctuations in dietary composition; reciprocally, changes in the microbiome have profound effects on animals, including piglets.

\section{Methods and materials Preparation of coix seed}

Coix seed was purchased from Donglan Medical Co., Ltd. The seed was then dried and ground into fine powder for direct mixing with feed. The extraction process of coix seeds was as follows: (1) Extract: the dry coix seed was added to the extraction tank, soaked in drinking water at six times the weight of the drug for $12 \mathrm{~h}$, and cooked twice (boiling, temperature $95-100{ }^{\circ} \mathrm{C}$ ).
Water was added at six times the drug weight and volume for $1.5 \mathrm{~h}$ each time, and the extract was filtered twice. (2) Concentration: the extract was concentrated at a relative density of 1.10 at $50{ }^{\circ} \mathrm{C}$ by mixing the filtrated extract collected in step 1. (3) Drying: dextrin ( $1 \%$ by weight) was added to the concentrated liquid, which was stirred evenly and dried by spraying; the temperature in the drying tower was $90-95^{\circ} \mathrm{C}$. (4) Sieve mixing: the spray-dried powder was passed through an $80-160$ mesh sieve, mixed evenly, and packaged to be used in the next step.

\section{Study animals and treatment}

In total, 40 weaned piglets (Duroc $\times$ Landrace $\times$ Yorkshire; male to female ratio $=1$ ), with an initial average body weight of $7.315 \pm 0.93 \mathrm{~kg}$ and weaned at $21 \pm 1$ day of age, were purchased from Guangming Farm and Animal Husbandry Co., Ltd. (Fujian, China). They were randomly and equally divided into four groups: the (i) control group, (ii) antibiotic group, (iii) coix seed powder group (TCMP), and (iv) coix seed extract group (TCME). The piglets were ear-labeled and their weights were recorded individually, followed by feeding with the experimental diets for 4 weeks. The basal diet was formulated to meet the NRC (2012) recommendations for the nutrient requirements of piglets. The ingredients and compositions of the basal diet are shown in Table 1. The piglets were maintained under standard light $(14 \mathrm{~h}$ light/dark) and temperature $\left(25 \pm 2{ }^{\circ} \mathrm{C}\right)$ conditions. The control group $(\mathrm{n}=10)$ was fed the basal diet. The piglets in the antibiotic group were provided fodder containing 1/10,000 amoxicillin powder for animals (antibiotics group, $\mathrm{n}=10$ ). The piglets in the coix seed powder group were given fodder containing $5 / 100$ coix seed powder (TCMP group, $n=10$ ). The piglets in the coix seed extract group were provided with fodder containing $1 / 100$ coix seed extract (TCME group, $n=10$ ).

\section{Table 1 Basic diet and nutrients}

\begin{tabular}{lc}
\hline Corn, \% & 50 \\
Wheat peel, \% & 4 \\
Soybean meal, \% & 30 \\
Fish meal, \% & 6 \\
Premix, \% & 10 \\
Total, \% & 100 \\
Digestible energy DE $\left(\mathrm{MJ} \mathrm{Kg}^{-1}\right)$ & 13.87 \\
Crude protein CP, \% & 19.5 \\
Lysine Lys, \% & 1.1 \\
Calcium Ca, \% & 0.9 \\
Available phosphorus AP, \% & 0.45 \\
\hline
\end{tabular}




\section{Calculation of average body weight and feed/meat ratio}

All piglets were weighed after overnight fasting on the morning of day 1 as well as on day 15 and day 28 of the feeding trial. The feed intake of each pig was recorded daily throughout the trial to calculate the feed/meat ratio (F:G).

\section{Determination of gut $\mathrm{pH}$ value and the gastrointestinal tract villi}

At the end of the 28-day trial, the piglets were sacrificed in accordance with the experimental animal procedures of the Institutional Ethics Committee/Animal Care and Use Committee, and the $\mathrm{pH}$ values of the stomach, duodenum, small intestine, colon, ileum, cecum, and rectum contents were measured using a pH meter (PHS-5C $\mathrm{PH}$, Guangzhou, China). Tissues $(1 \mathrm{~cm})$ from the stomach, duodenum, small intestine, colon, ileum, cecum, and rectum were collected from the piglets and fixed for $12 \mathrm{~h}$ in $5 \mathrm{~mL} 4 \%$ paraformaldehyde. The fixed tissue was sequentially passed through $50 \%, 70 \%, 80 \%, 90 \%, 100 \%$, and $100 \%$ alcohol for 35 to $45 \mathrm{~min}$ at each concentration and clarified using $100 \% \mathrm{C}_{2} \mathrm{H}_{5} \mathrm{OH}+$ xylene (1:1) for 30 to $40 \mathrm{~min}$. The tissues were then immersed in paraffin (1:1) for $30 \mathrm{~min}$. Sections of 6-12 $\mu \mathrm{m}$ thickness were cut (average $7-8 \mu \mathrm{m}$ ), placed onto slides, and stained with hematoxylin and eosin. The sections were observed and villus length was measured under a microscope.

\section{Sample collection and extraction of genomic DNA from gastrointestinal microorganisms}

The contents of the stomach, duodenum, small intestine, colon, ileum, cecum, and rectum were collected from the piglets, and total genomic DNA from these samples was extracted using the CTAB/SDS method. The DNA concentration and purity were monitored on $1 \%$ agarose gels. The DNA was diluted to $1 \mathrm{ng} / \mu \mathrm{L}$ using sterile water.

\section{PCR and sequencing of gastrointestinal microorganisms}

The 16s rRNA sequences of the gastrointestinal microorganisms were amplified using the specific primer for 16S v3-4: ACTCCTACGGGAGGCAGCA and GGA CTACHVGGGTWTCTAAT (MK351252.1). The raw sequencing data were uploaded into the NCBI Sequence Read Archive database (SRA; http://www.ncbi.nlm.nih. gov/Traces/sra/) under accession number SRP156563. All PCR were carried out with $25 \mu \mathrm{L}$ sample volumes and $12.5 \mu \mathrm{L}$ of Phusion ${ }^{\circledR}$ High-Fidelity PCR Master Mix (New England Biolabs), $0.2 \mu \mathrm{mol}$ forward and reverse primers, and $10 \mathrm{ng}$ template DNA. Thermal cycling was started with initial denaturation at $98{ }^{\circ} \mathrm{C}$ for 10 min followed by 35 cycles of denaturation at $98{ }^{\circ} \mathrm{C}$ for $10 \mathrm{~s}$, annealing at $50{ }^{\circ} \mathrm{C}$ for $40 \mathrm{~s}$, and elongation at $72{ }^{\circ} \mathrm{C}$ for $50 \mathrm{~s}$, and a final elongation at $72{ }^{\circ} \mathrm{C}$ for $10 \mathrm{~min}$.

\section{Library preparation and sequencing}

Sequencing libraries were generated using an NEB Next ${ }^{\circledR}$ Ultra $^{\mathrm{TM}}$ DNA Library Prep Kit for Illumina (NEB, USA) following the manufacturer's recommendations, and index codes were added. The library quality was assessed on a Qubit@2.0 Fluorometer (Thermo Scientific) and an Agilent Bioanalyzer 2100 system. Finally, the library was sequenced on an Illumina HiSeq 2500 platform and $250 \mathrm{bp}$ paired-end reads were generated.

\section{Data analysis}

Sequence analysis was performed with the UPARSE software package using the UPARSE-OTU and UPARSEOTUref algorithms. In-house Perl scripts were used to analyze the alpha (within samples) and beta (among samples) diversity. Sequences with $\geq 97 \%$ similarity were assigned to the same OTUs. We selected representative sequences for each OTU and used an RDP classifier to annotate the taxonomic information for each representative sequence. In order to compute the alpha diversity, we rarified the OTU table and calculated three metrics: Chao1, which estimates the species abundance; observed species, which estimates the number of unique OTUs found in each sample; and Shannon index. Rarefaction curves were generated based on these three metrics.

\section{Community distribution}

A graphical representation of the relative abundance of bacterial diversity from the phylum to species level can be visualized using a Krona chart. Cluster analysis was preceded by principal component analysis (PCA), which was applied to reduce the dimension of the original variables using the QIIME software package. QIIME can calculate both weighted and unweighted UniFrac distances, which are phylogenetic measures of beta diversity. We used unweighted UniFrac distances for principal coordinate analysis (PCoA) and the unweighted pair group method with arithmetic mean (UPGMA) clustering. PCoA helps to obtain principal coordinates and visualize them from complex, multidimensional data. The maximum variation factor is demonstrated by the first principal coordinate, the second-largest one is demonstrated by the second principal coordinate, and so on. UPGMA clustering is a hierarchical clustering method that uses average linkage and can be used to interpret the distance matrix.

\section{Statistical analysis}

To confirm the differences in the abundances of individual taxae between groups, Metastats software (Version:1.0) was utilized. LEfSe was used for the quantitative analysis of biomarkers within different groups. This method was designed to analyze data in which the number of species is much higher than the number of samples 
and to provide biological class explanations to establish statistical significance, biological consistency, and effectsize estimation of the predicted biomarkers. To identify the differences in microbial communities between groups, ANOSIM and MR PP (multi-response permutation procedure) were performed based on the Curtis dissimilarity distance matrices.

\section{Results}

\section{Growth performance}

The average body weights of piglets are shown in Fig. 1. Body weight on day 28 was found to have increased under diets containing coix seed and antibiotics $(p<0.05)$. Meanwhile, the TCMP group on day 14 showed higher body weight in pigs fed diets with coix seed powder than that of pigs fed diets with either antibiotics or coix seed extracts $(p<0.05)$. However, on day 28 , the highest body weight was measured in the TCME group. In addition, a lower F:G was observed in pigs fed a coix seed diet as compared to those on the antibiotic diet and control diets $(p<0.05)$.

\section{Gastrointestinal $\mathrm{pH}$ value}

The $\mathrm{pH}$ value of the gastrointestinal tract is closely related to the digestion and absorption of nutrients. It affects the microenvironment for the growth of gastrointestinal microbiota and plays an important role in regulating microbial growth. In this study, we found that the $\mathrm{pH}$ value of the succus gastricus in the coix seed group (TCMP and TCME) was between 2 and 3 (Fig. 2), and was lower than those of the antibiotic group and control group $(p<0.05)$. This is also the optimal activation condition for pepsin A and pepsin B. Moreover, compared with the control group and antibiotic group, the $\mathrm{pH}$ values of the colon, rectum, and cecum in the coix seed powder group significantly increased on day $28(p<0.05)$.

\section{Morphological changes in gastrointestinal villi in piglets}

The results of the gastric villus morphology assay are shown in Fig. 3. On day 28, the villus height was more in piglets fed a coix seed diet (TCP and TCME) than in piglets fed an antibiotic diet or control diet $(p<0.05)$. Villus height in the colon, ileum, and cecum also clearly increased in piglets on a coix seed diet compared to that in piglets fed a normal diet or an antibiotic diet (Fig. 4). The maximum villus height was observed in piglets fed coix seed extract. In addition, there were some differences in villus height between the antibiotic group and control group on day 28.

\section{Coix seed treatment altered the gut microbiota of weaning piglets}

In order to investigate the effect of coix seed on the composition of gut microbiota, we analyzed the bacterial populations of the succus gastricus, colon, ileum, and cecum on day 14 and day 28 . The weaning pigs fed a coix seed diet showed a high relative abundance of the genus Bactobaccillus and phylum Firmicutes in the succus gastricus as compared to the other groups, with a significant increase from day 14 to day 28 (Fig. 5). Meanwhile, pigs supplemented with the extract of coix seed showed a clear decrease in abundance of genus Prevotellaceae and genus Lactobacillus in the ileum. The antibiotic group and control group showed no changes in genus Prevotellaceae in the ileum; simultaneously, the genus Lactobacillus in the ileum of both the antibiotic group and control group declined from day 14 to day 28 (Fig. 6). In weaning pigs fed with a coix seed diet, both genus and phylum Bacteroides in the colon showed higher abundance
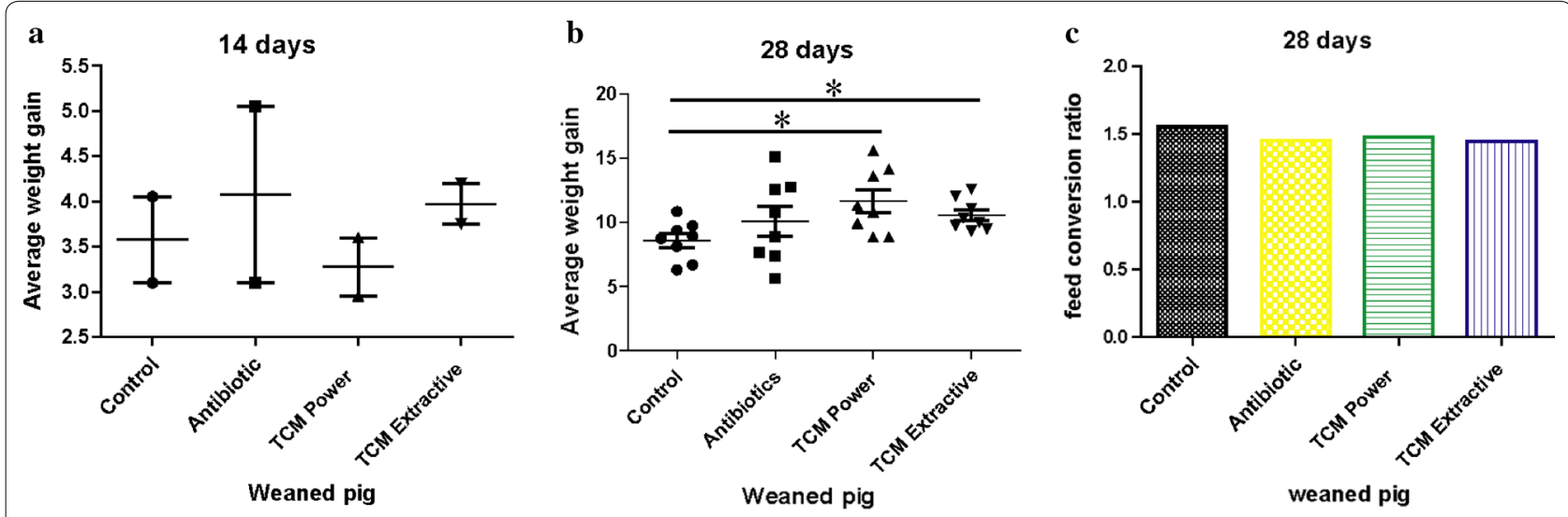

Fig. 1 Average weight gain and feed/meat ratio of weaned pigs. a Average weight gain of weaned pigs at 14 days after the start of the feeding experiment. $\mathbf{b}$ Average weight gain at 28 days after the start of the feeding experiment. c Feed/meat ratio of weaned pigs at 28 days after the start of the feeding experiment. $\left({ }^{*} p<0.05\right)$ 


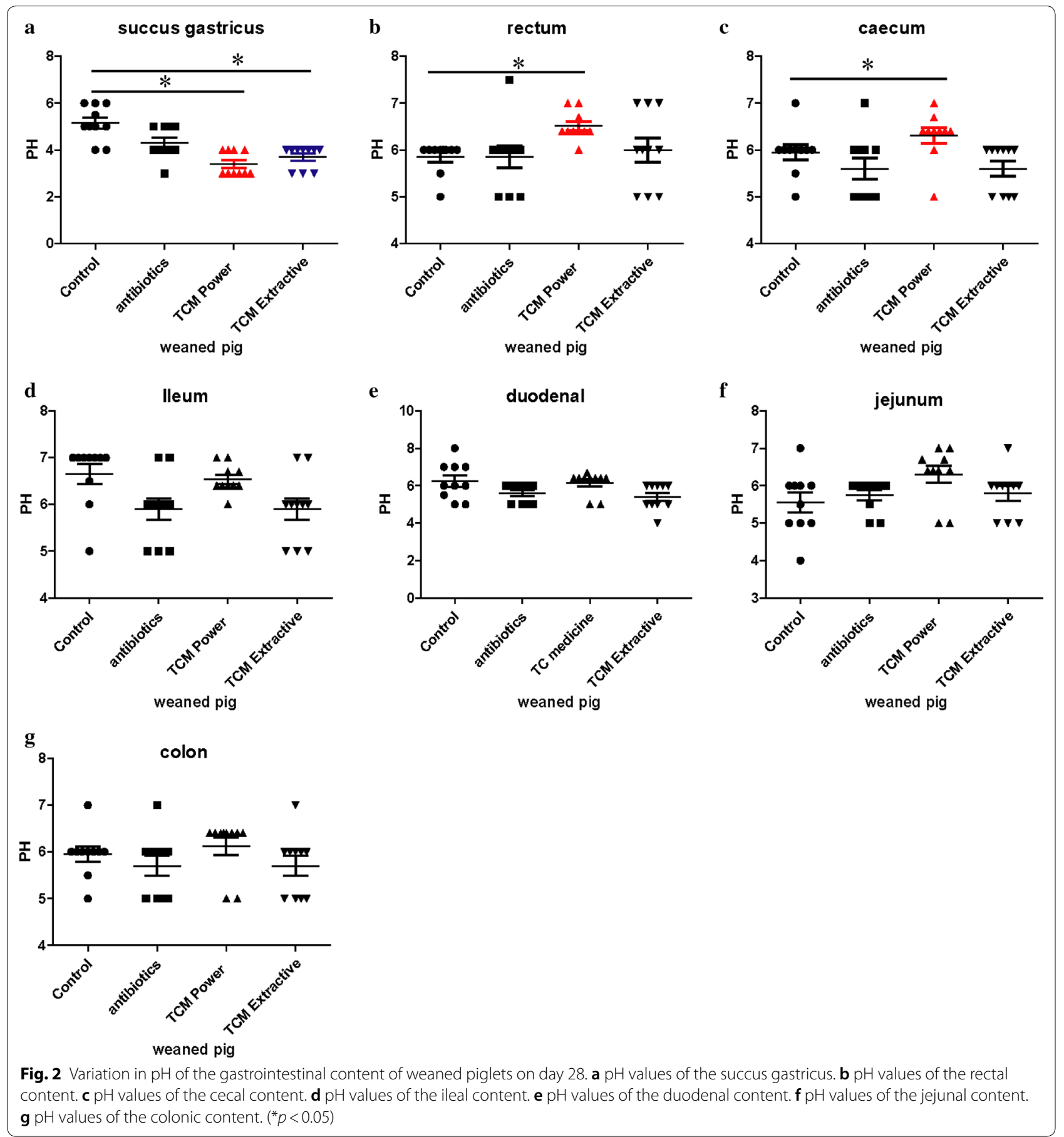

as compared to that in the antibiotic group and control group. From day 14 to 28, the genus Bacteroides in the colon showed an increase in TCMP and TCME, but was significantly decreased in the other groups (Fig. 7). We also observed that treatment with coix seed decreased the abundance of genus Prevotella and increased the abundance of genus Bacteroides in the cecum (Fig. 8).

\section{Discussion}

Here, we demonstrated for the first time that feeding weaning pigs a coix seed diet can result in significantly improved growth and a reduced F:G. The coix seed diet also significantly reduced the $\mathrm{pH}$ of the succus gastricus, increased villus length, and significantly modulated the composition of gut microbiota. 

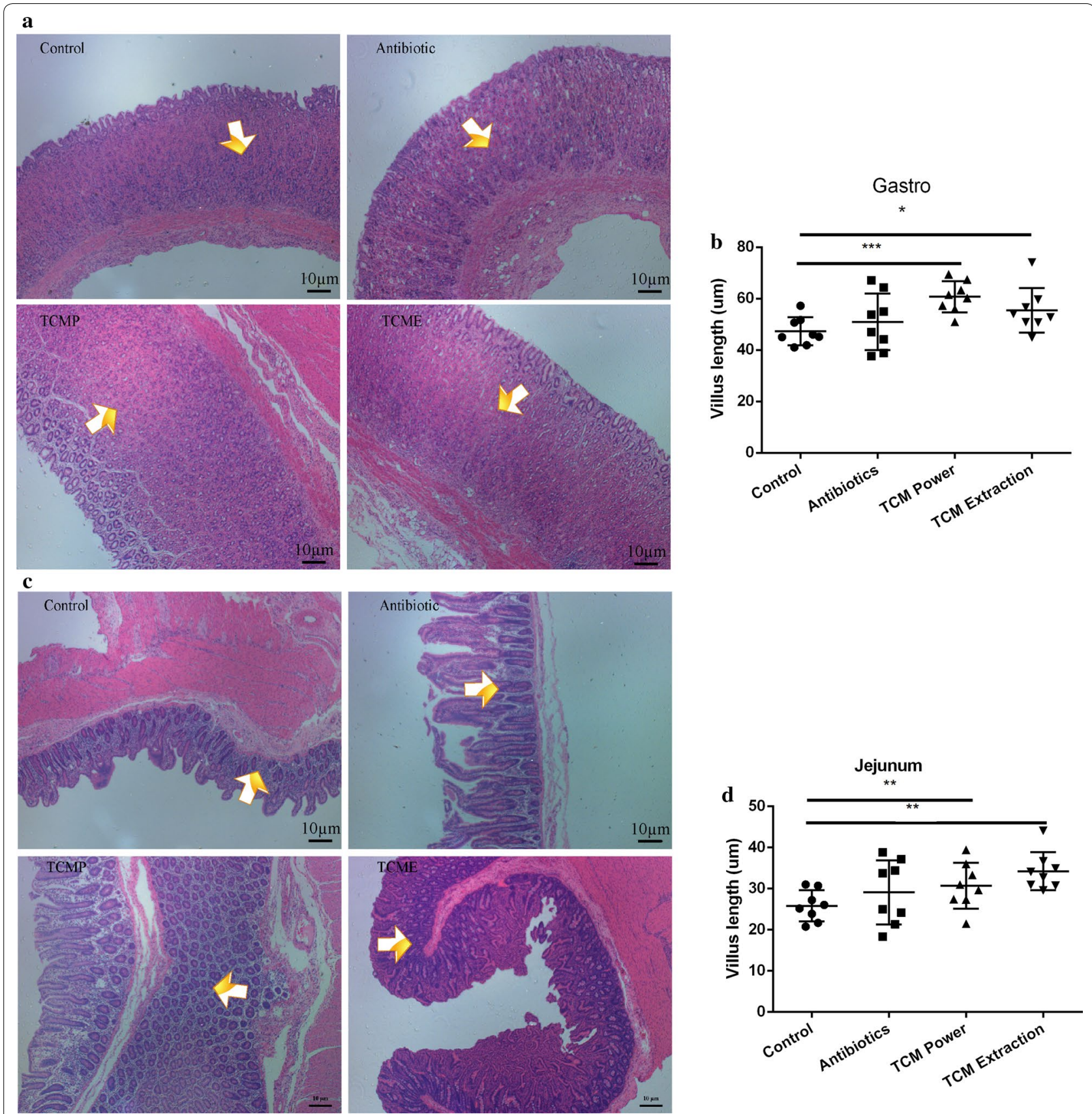

Fig. 3 Morphological changes in the gastrointestinal villi of weaned pigs. a Morphological changes in gastric villi in weaned pigs. $\mathbf{b}$ Length of the gastric villi. c Morphological changes in the jejunum of weaned pigs. $\mathbf{d}$ Length of jejunal villi in different groups. ${ }^{*} p<0.05 ;{ }^{* *} p<0.02 ;{ }^{* * *} p<0.01$ )

Weaning pigs often are exposed to a multitude of stressors such as diet conversion, littermate separation, and disturbances in the surrounding environment, which frequently cause intestinal dysfunction, pathogen invasion, appetite decrease, feed intake decline, microecological imbalance, and growth retardation (Blecha and Kelley 1981; Funderburke and Seerley 1990; Ahrens et al. 2009; Hyun et al. 1998; Moeser et al. 2017). Therefore, maintaining normal gut health and microecological balance is vital to the growth performance of weaned pigs (Lallès et al. 2002). At present, antibiotics and additives have been widely used to alleviate the negative effects of weaning stressors (Lucas et al. 1959; Choi et al. 2011; Stahly et al. 1981). However, misuse and overuse of antibiotics increase antimicrobial resistance, which poses a serious threat of growing concern to human, animal, 

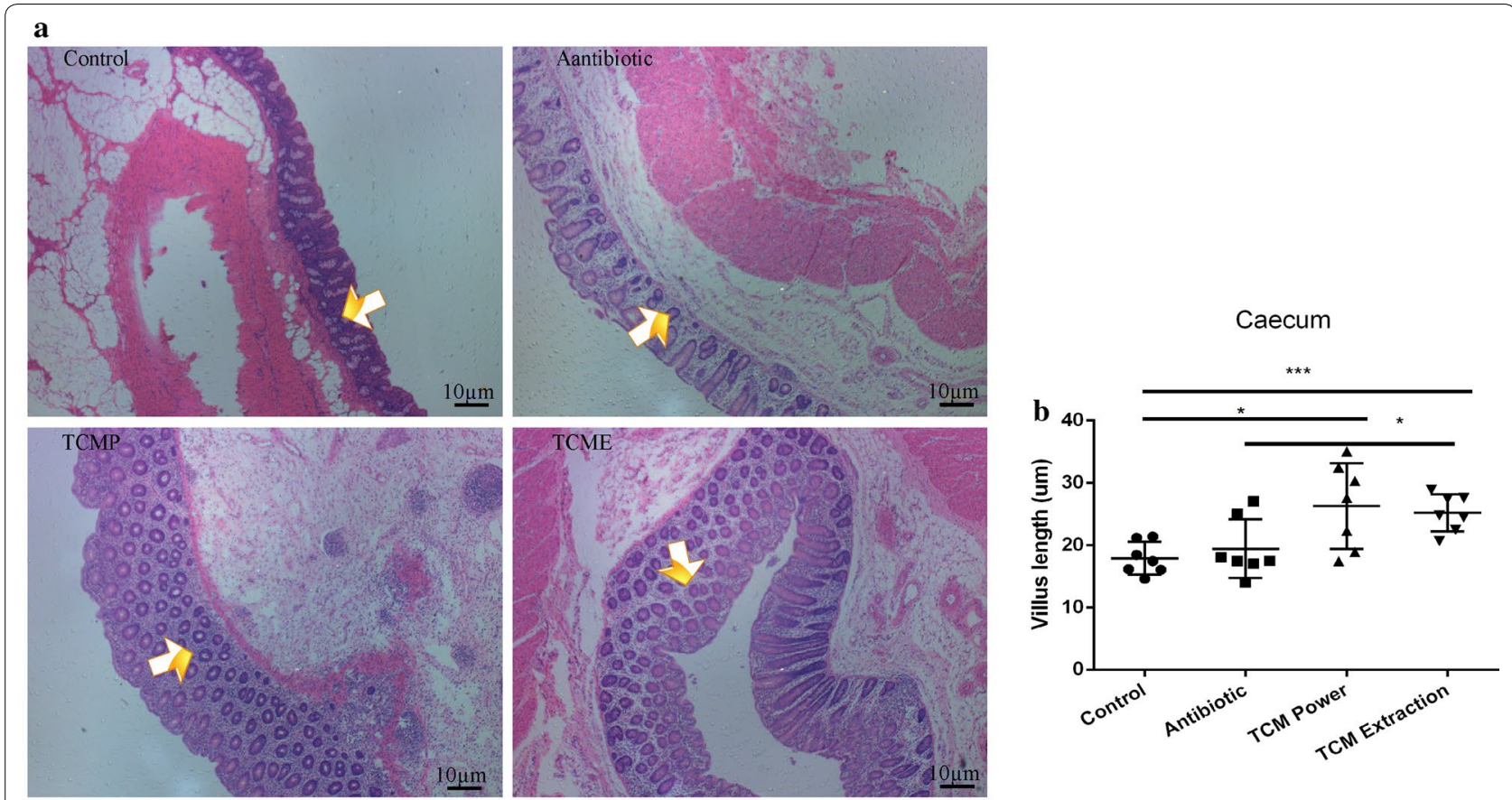

c
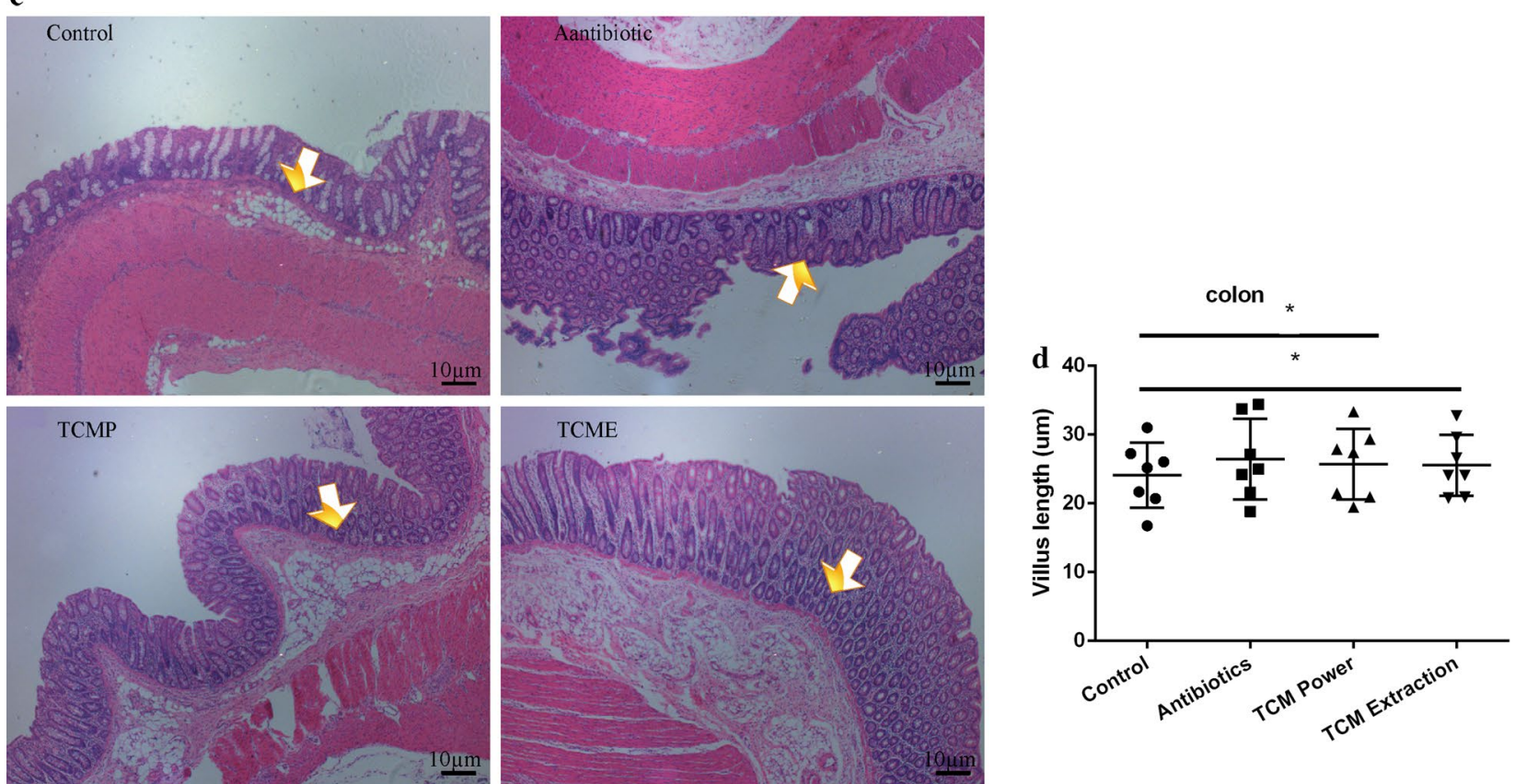

Fig. 4 Morphological changes in the cecal and colonic villi of weaned pigs. a Morphological changes in the ceca of weaned pigs; $\mathbf{b}$ Length of the cecal villi. c Morphological changes in the colons of weaned pigs. $\mathbf{d}$ Length of colonic villi in different groups. $\left({ }^{*} p<0.05 ;{ }^{* * *} p<0.01\right)$

and environmental health (Hamscher et al. 2003; Sapkota et al. 2006; Murphy et al. 2007; Ferguson et al. 2016). Therefore, some herbal TCMs have been considered as substitutes for antibiotics. Rhizoma Coptidis, Rhizoma Atractylodis, Pericarpium Citri Reticulatae, Fructus Mume, Fructus Psoraleae, Poria Cocos, and Semen
Lablab Albumis are commonly used for their antidiarrheal or antimicrobial activity (Wang et al. 2017; Dong et al. 2007; Zhang 2005; Geng et al. 2011; Cheng et al. 2017; Fuchs et al. 2006). Coix seed is a plant material with considerable anti-insufficiency of spleen yang, anti-cancer, and anti-inflammatory properties (Han et al. 

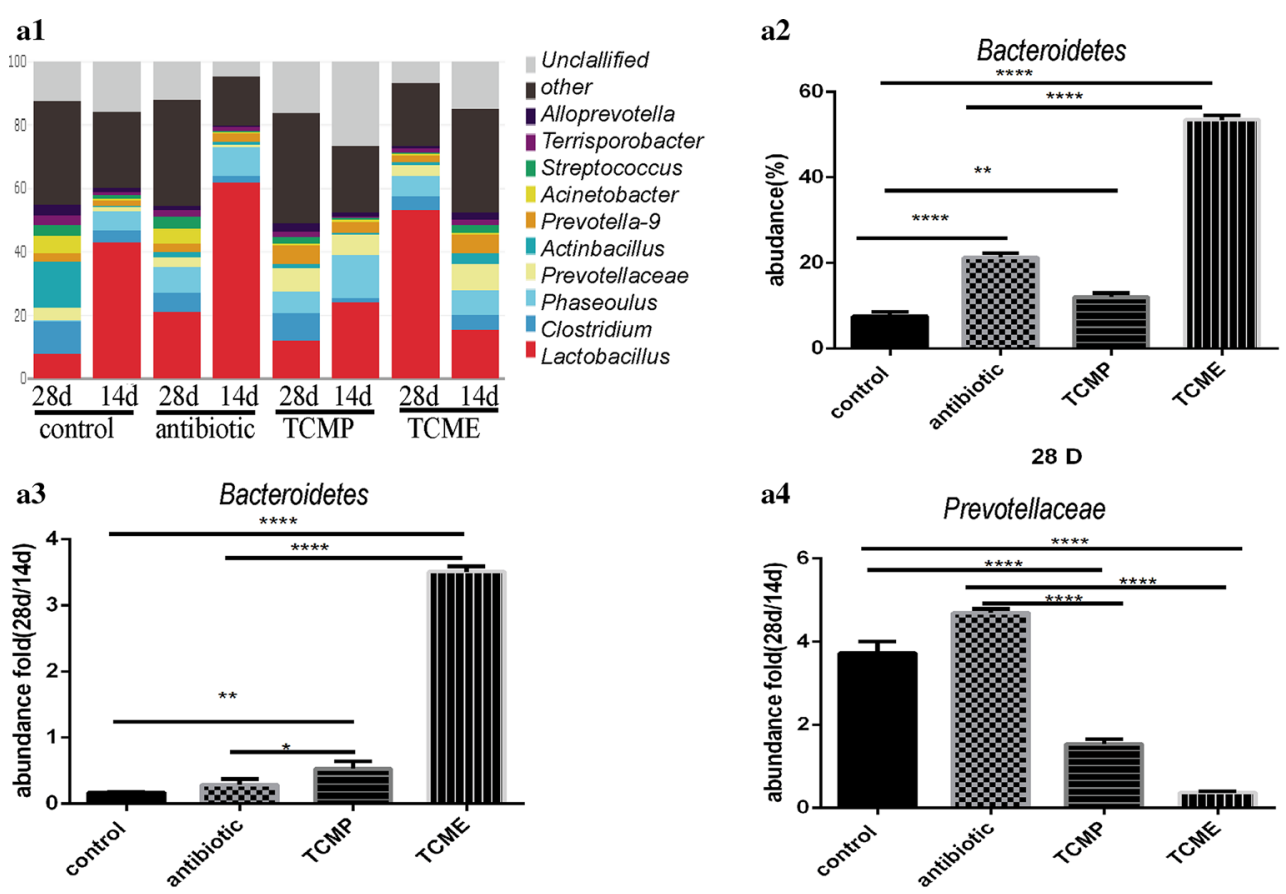

Genus

$28 \mathrm{D}$

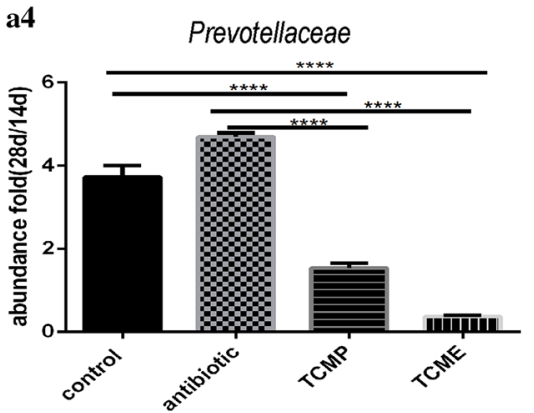

Genus

b1

b2
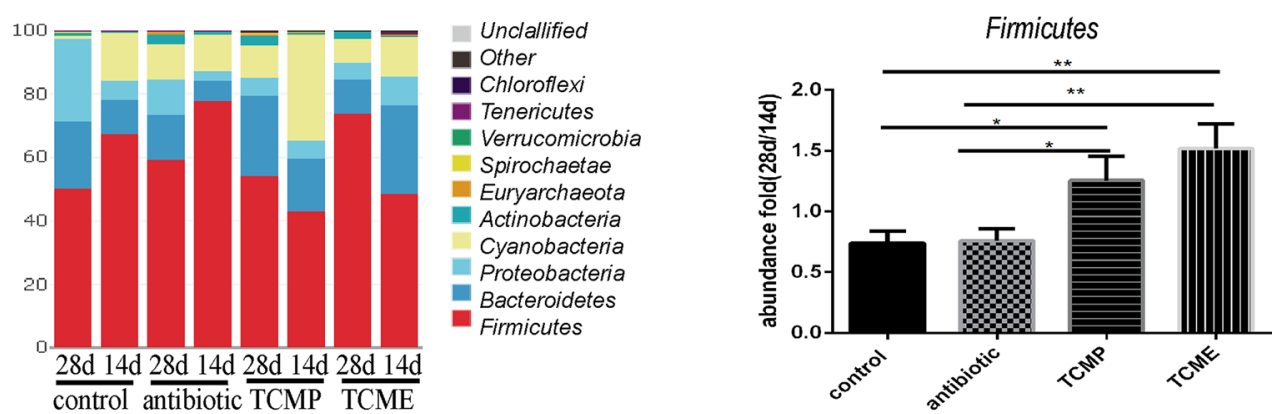

Phylum

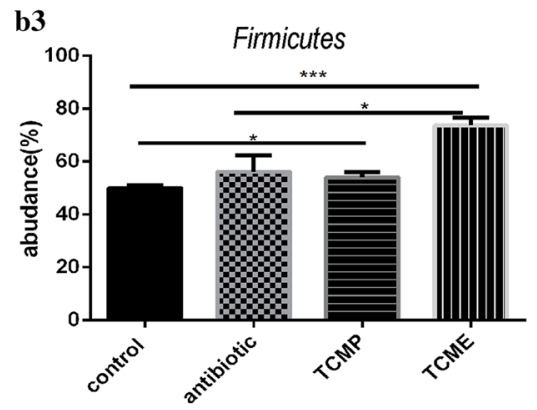

Phylum(28d)

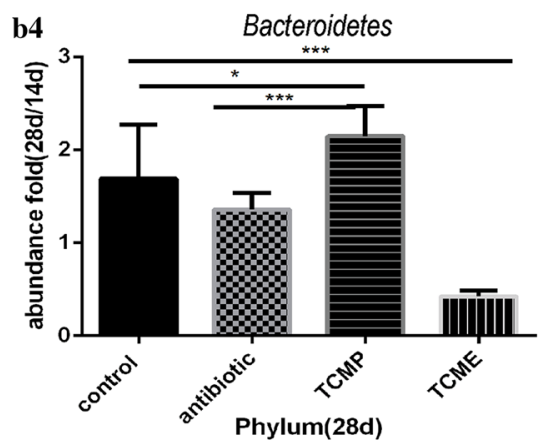

Fig. 5 Effect of coix seed on the composition of gastrointestinal microbiota with respect to genus and phylum. a1 Effect of coix seed on the composition of the gastrointestinal microbiota with respect to genus in weaned pigs. a2 Abundance of genus Bacteroidetes. a3 Fold abundance of genus Bacteroidetes (28D/14D). a4 Fold abundance of genus Prevotellaceae (28D/14D). b1 Effect of coix seed on the composition of the gastrointestinal microbiota with respect to phylum in weaned pigs. b2 Fold abundance of phylum Firmicutes (28D/14D). b3 Abundance of phylum Firmicutes. (b4) Fold abundance of phylum Bacteroidetes (28D/14D). ${ }^{*} p<0.05 ;{ }^{* *} p<0.02 ;{ }^{* * *} p<0.01$, ${ }^{* * *} p<0.001$ ) 

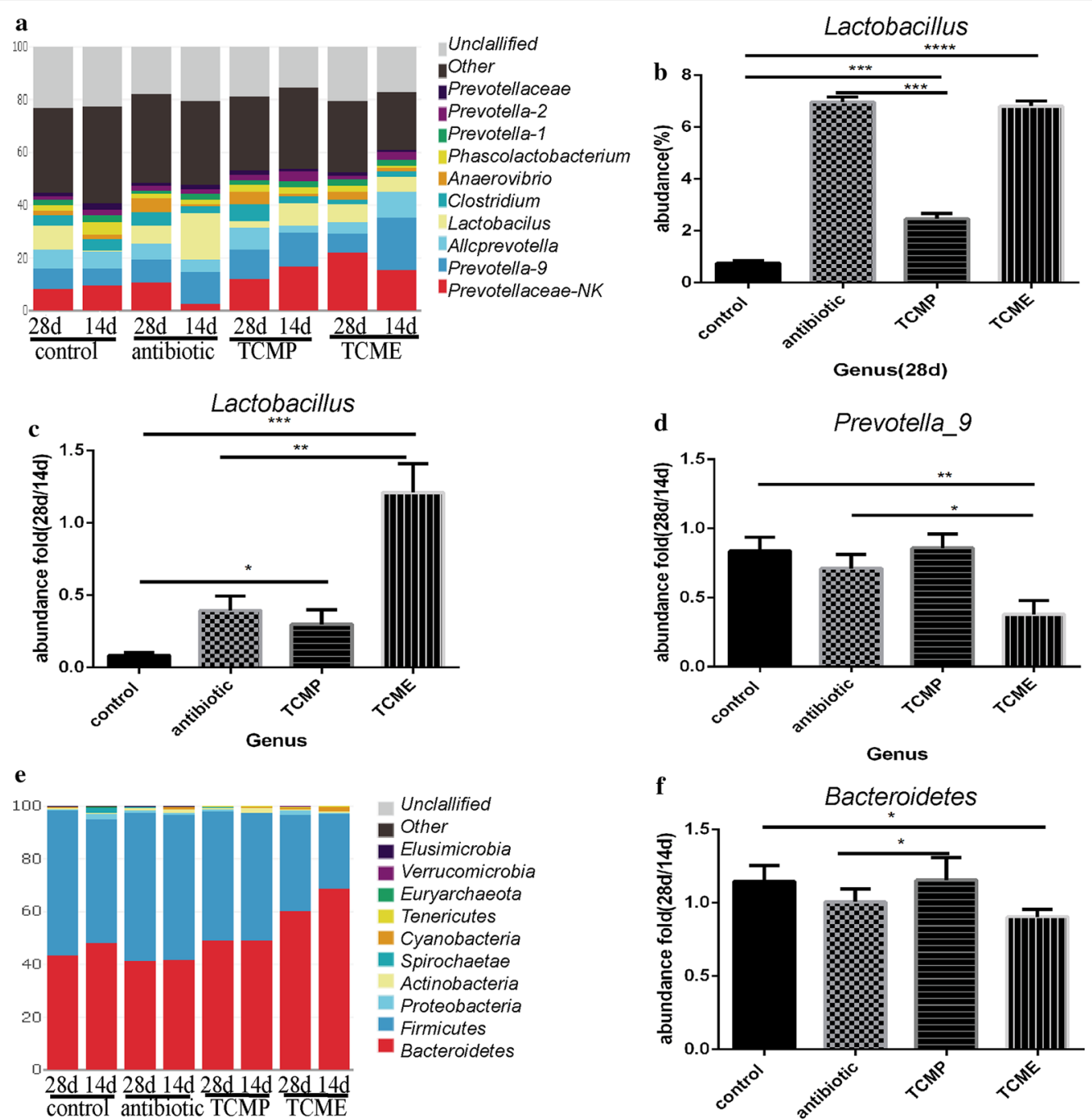

Phylum

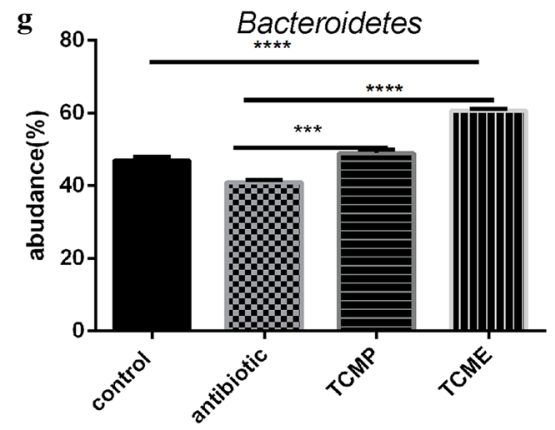

Phylum(28d)

h

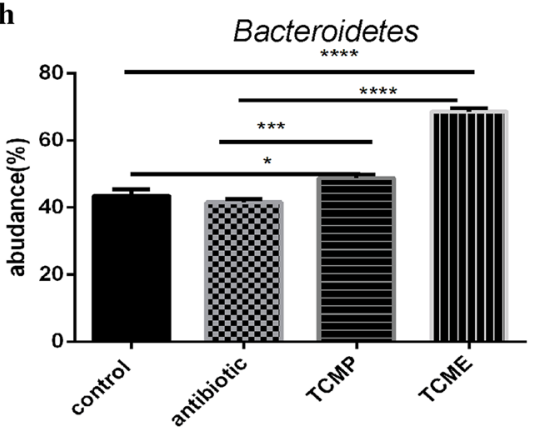

Phylum(14d)

Fig. 6 Effect of coix seed on the composition of cecal microbiota with respect to genus and phylum. a1 Effect of coix seed on the composition of cecal microbiota with respect to genus in weaned pigs. a2 Abundance of genus Lactobacillus. a3 Fold abundance of genus Lactobacillus (28D/14D). a4 Fold abundance of genus Prevotella-9 (28D/14D). b1 Effect of coix seed on the composition of cecal microbiota with respect to phylum in weaned pigs. b2 Fold abundance of phylum Bacteroidetes (28D/14D). b3 Abundance of phylum Bacteroidetes. b4 Fold abundance of phylum Bacteroidetes (14D) $\left(^{*} p<0.05 ;{ }^{* *} p<0.02 ;{ }^{* * *} p<0.01,{ }^{* * *} p<0.001\right)$ 

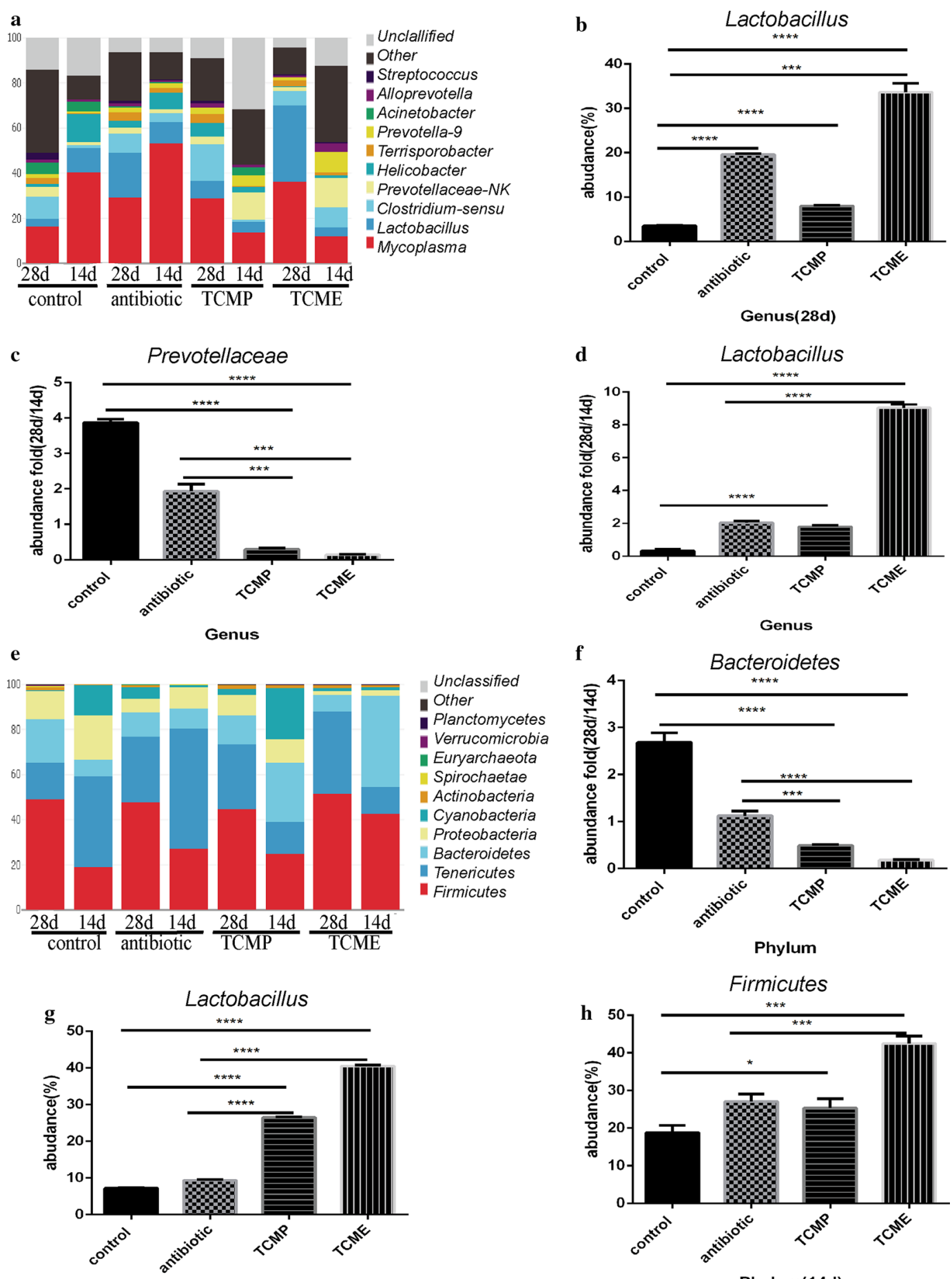

Phylum

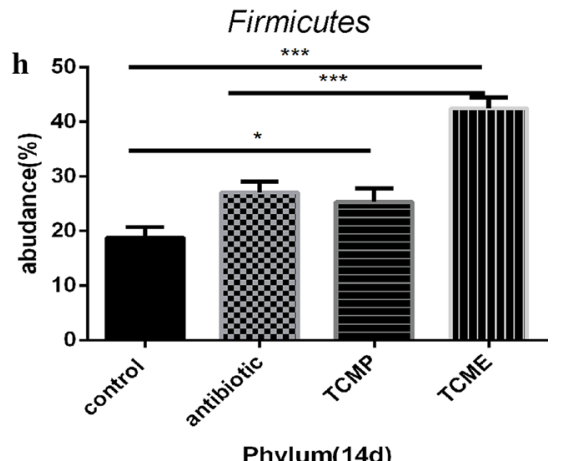

Phylum(14d)

Phylum(14d)

Fig. 7 Effect of coix seed on the composition of jejunal microbiota with respect to genus and phylum. a Effect of coix seed on the composition of jejunal microbiota with respect to genus in weaned pigs. b Abundance of genus Lactobacillus. c Fold abundance of genus Prevotellaceae (28D/14D). d Fold abundance of genus Lactobacillus (28D/14D). e Effect of coix seed on the composition of jejunal microbiota with respect to phylum in weaned pigs. f Fold abundance of phylum Bacteroidetes (28D/14D). $\mathbf{g}$ Abundance of phylum Lactobacillus. $\mathbf{h}$ Fold abundance of phylum Firmicutes (14D). $\left({ }^{*} p<0.05 ;{ }^{* *} p<0.02 ;{ }^{* * *} p<0.01,{ }^{* * *} p<0.001\right)$ 

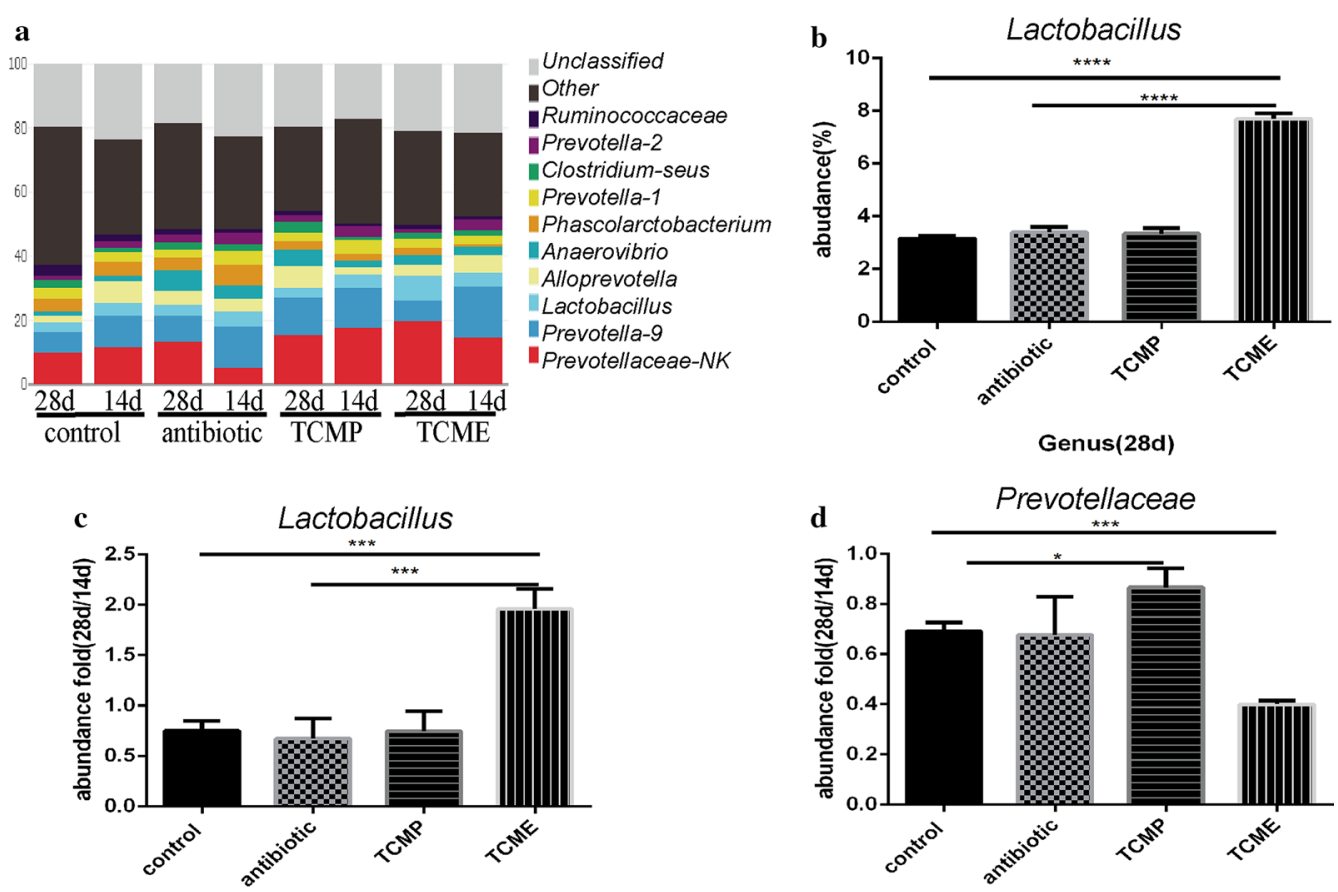

Genus

Genus

e
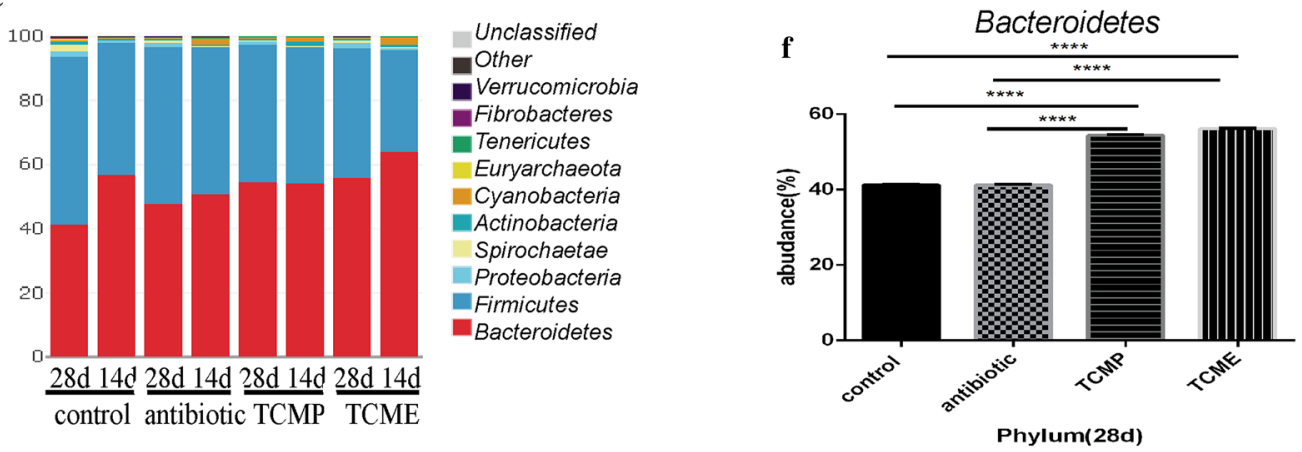

Unclassified Other Verrucomicrobia - Fibrobacteres Tenericutes

Euryarchaeota Cyanobacteria Actinobacteria Spirochaetae

Proteobacteria Firmicutes Bacteroidetes control antibiotic TCMP TCME

Phylum(28d)

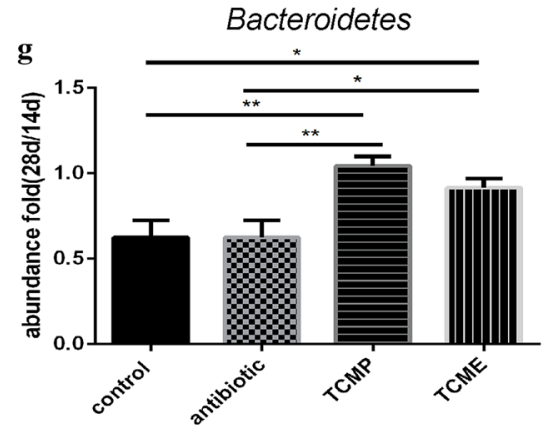

Phylum

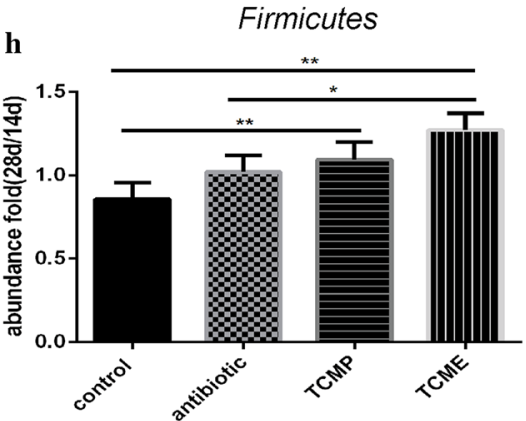

Phylum

Fig. 8 Effect of coix seed on the composition of colonic microbiota with respect to genus and phylum. a Effect of coix seed on the composition of colonic microbiota with respect to genus in weaned pigs. b Abundance of genus Lactobacillus. c Fold abundance of genus Lactobacillus (28D/14D). d Fold abundance of genus Prevotellaceae (28D/14D). e Effect of coix seed on the composition of colonic microbiota with respect to phylum in weaned pigs. f Fold abundance of phylum Bacteroidetes (28D/14D). g Abundance of phylum Lactobacillus. h Fold abundance of phylum Firmicutes (14D). ( $\left.^{*} p<0.05 ;{ }^{* *} p<0.02 ;{ }^{* * *} p<0.01,{ }^{* * *} p<0.001\right)$ 
animal feed has shown some growth-promoting effects in cows (Luo et al. 2016), but the underlying mechanism has not been reported. Our results showed that coix seed actually improved growth performance and productivity post weaning. Furthermore, we found that coix seed significantly reduced the $\mathrm{pH}$ of the succus gastricus. Gastric acidity in piglets is known to promote the secretion and activity of digestive enzymes and plays a vital role in the process of piglet growth. Suckling piglets have relatively constant gastric acidity due to lactose fermentation, and thus dietary $\mathrm{pH}$ has little effect on their gastric $\mathrm{pH}$. After weaning, the pigs lack a supply of lactose, which results in increased gastric $\mathrm{pH}$, leading to gastrointestinal dysfunction and greater opportunity for pathogenic bacterial colonization. We found that a coix seed feed diet stabilized the gastric acidity in weaning pigs ( $\mathrm{pH} 2-3.5)$, providing optimal activation conditions for pepsin in gastric juice (Bednarzewski 1968; de Gara et al. 1986). This increased activity of gastrointestinal digestive enzymes in weaned pigs further promotes the digestion and absorption of nutrients and improves growth performance and productivity. Additionally, lower gastrointestinal $\mathrm{pH}$ provides a favorable growth environment for Lactobacillus (Ramos et al. 2014). In our study, the coix seed group showed greater abundance of the genus and phylum Lactobacillus as compared with the antibiotic and control groups, whereas the abundance of genus Prevotellaceae in the ileum was low. Therefore, we conclude that coix seed might reduce gastric $\mathrm{pH}$ and promote pepsin secretion and the growth of Lactobacillus in piglets. However, the specific mechanisms require further research.

The integrity of gastrointestinal villus morphology is the premise of animal health, because it plays a critical role in nutrient digestion, absorption, and resistance to the invasion of pathogenic bacteria (Yang et al. 2013; Chen et al. 2008). However, at weaning, a carbohydratebased diet damages the integrity of intestinal mucosa in piglets. In our control group and antibiotic group, the gastrointestinal villi showed emerging atrophy and disruption. This can largely be explained by the gastrointestinal epithelial cell damage and shedding caused by weaning. However, in the coix seed groups (TCMP and TCME), the gastrointestinal villi showed greater integrity and length. It is possible that the rich active components of esters, unsaturated fatty acids, sugars, and lactams in coix seed afforded sufficient nutrition for the growth of the intestinal villus epithelial cells.

It is well-established that gut microbiota affect host metabolism, induce metabolic disease, and play an important role in the health of animals and humans (Velagapudi et al. 2010; Cani et al. 2013). Gut microflora perform a wide variety of metabolic transformations, in which a variety of phytochemical compounds can be metabolized into products required for physiological activity (Eyssen 1973; Goldin 1990; Frick et al. 2012). Most oral medicines unavoidably affect gut microorganisms; however, traditional Chinese medicines are widely accepted as high-efficiency and low-toxicity "medicinal diets" that are capable of avoiding certain side effects and can be used to treat gastrointestinal discomforts by regulating microbiological balance (Chen et al. 2015). In this study, weaned pigs fed a coix seed diet showed a significant increase in Lactobacillus and Bacteroides in the gastrointestinal system, along with a decline in the abundance of Prevotellaceae. Because coix is similar to other cereals, starch is its main component, accounting for approximately $60 \%$, and the content of oil, polysaccharides, and protein is higher than that in conventional cereals like rice and wheat; this makes coix a good culture medium for gut microbiota (Yang et al. 2011). In addition, the components of proteins and polysaccharides in coix seed play an important role in regulating water transport and afford a good growth environment for gut microbiota.

Previous studies have demonstrated that coix seed can increase the abundance of Lactobacillus and Coprococcus, induce imbalance of intestinal microbiota, and increase levels of probiotic bacteria (Caesar et al. 2015). Another report showed that coix seed enriches microbial metabolic pathways such as glycerolipid metabolism, biosynthesis of unsaturated fatty acids, the sulfate transport system, the manganese/iron transport system, and the glutathione transport system (Liu et al. 2019). Therefore, coix seed is a useful dietary supplement for the treatment of imbalanced gut microbial ecology (Wang et al. 2015). Thus, we speculated that coix seed would improve growth and reduce F:G in weaned pigs, which could be associated with increased amounts of Lactobacillus and Bacteroides and the enrichment of microbial metabolic pathways.

Collectively, the present study provides the first evidence that coix seed has beneficial effects on intestinal villus development and intestinal barrier function in weaned pigs, which might partly explain why the growth performance of pigs was improved by dietary coix seed supplementation.

\section{Authors' contributions \\ $Z L L, Z N L$, and $Z Y Y$ designed experiments, and ZLL and ZNL directed experi- ments and wrote the manuscript. ZLL and ZNL performed experiments. $Z L$, ZHF, QC, SFD, ZWL, and YQY helped with the experimentation. ZLL, ZNL, and $\mathrm{ZYY}$ analyzed data and revised the manuscript. All authors read and approved the final manuscript.}

Funding

This study was funded by the Central Guidance for the Special Development of Local Science and Technology (2017L3006), the China Agriculture Research 
System (CARS-22), and the Scientific and Technological Innovation Team of FAAS, China (STIT2017-1-9)

\section{Availability of data and materials}

All data generated or analyzed during this study are included in this published article

\section{Ethics approval and consent to participate}

All procedures performed in studies involving animal participants were in accordance with the ethical standards of the Fujian Academy of Agricultural Sciences Research Committee and with the 1964 Helsinki Declaration and its later amendments or comparable ethical standards.

\section{Consent for publication}

Provided during the submission process. Not applicable.

\section{Competing interests}

The authors declare that they have no competing interests.

\section{Author details}

${ }^{1}$ Fujian Key Laboratory of Innate Immune Biology, Biomedical Research Center of South China, Fujian Normal University Qishan Campus, Fuzhou 350117, Fujian, China. ${ }^{2}$ Institute of Animal Husbandry and Veterinary Medicine, Fujian Academy of Agricultural Sciences, Pudang, Jin-an District, Fuzhou 350013, Fujian, China. ${ }^{3}$ Agricultural Ecology Institute, Fujian Academy of Agricultural Sciences, Pudang, Jin-an District, Fuzhou 350013, Fujian, China. ${ }^{4}$ Fujian Engineering and Technology Research Center for Hilly Prataculture, Pudang, Jin-an District, Fuzhou 350013, Fujian, China.

Received: 24 January 2019 Accepted: 1 July 2019

Published online: 23 July 2019

\section{References}

Ahrens F, Sünkel Y, Pollmüller T, Bussemas R, Weißmann F, Erhard M (2009) Influence of two different stressors, weaning and immunization, on the plasma histamine level of organic farming piglets. Inflamm Res 58(Suppl 1):53-54. https://doi.org/10.1007/s00011-009-2005-3

Bao K (2017) Non-scientific classification of Chinese herbal medicine as dietary supplement. Chin J Integr Med. https://doi.org/10.1007/s1165 5-016-2536-8

Bednarzewski J (1968) Studies on the correlation between gastric acidity determined by titration and $\mathrm{pH}$-metry and pepsin activity of the gastric juice, blood plasma and urine. Pol Tyg Lek 23:126-129

Blecha F, Kelley KW (1981) Effects of cold and weaning stressors on the antibody-mediated immune response of pigs. J Anim Sci 53:439-447. https://doi.org/10.2527/jas1981.532439x

Caesar R, Tremaroli V, Kovatcheva-Datchary P, Cani P, Bäckhed F (2015) Crosstalk between gut microbiota and dietary lipids aggravates wat inflammation through TLR signaling. Cell Metab 22:658-668

Cani P, Everard A, Duparc T (2013) Gut microbiota, enteroendocrine functions and metabolism. Curr Opin Pharmacol 13:935-940. https://doi. org/10.1016/j.coph.2013.09.008

Centner T (2016) Recent government regulations in the United States seek to ensure the effectiveness of antibiotics by limiting their agricultural use. Environ Int 94:1-7. https://doi.org/10.1016/j.envint.2016.04.018

Chen X, Zhao J, Gregersen $\mathrm{H}$ (2008) The villi contribute to the mechanics in the guinea pig small intestine. Fed Am Soc Exp Biol J 41:806-812

Chen D-K, Li L-L, Zhang B, Zhang F-S, Li H-Q, Jiang J-J (2009) Effects of different additives on cherry volley duck growth performance and duck housemephitis concentration. Chinese J Ecol 28:1499-1504

Chen F, Wen Q, Jiang J, Li H-L, Tan Y, Li Y-H, Zeng N-K (2015) Could the gut microbiota reconcile the oral bioavailability conundrum of traditional herbs? J Ethnopharmacol 179:253-264. https://doi.org/10.1016/j. jep.2015.12.031

Cheng X, Huo J, Wang D, Cai X, Sun X, Lu W et al. (2017) Herbal medicine AC591 prevents oxaliplatin-induced peripheral neuropathy in animal model and cancer patients. Front Pharmacol 8:344

Choi JY, Shinde PL, Ingale SL, Kim J, Kim YW, Kim KH, Kwon IK, Chae BJ (2011) Evaluation of multi-microbe probiotics prepared by submerged liquid or solid substrate fermentation and antibiotics in weaning pigs. Livest Sci LIVEST SCI 138:144-151. https://doi.org/10.1016/j.livsci.2010.12.015

Coffin R, Lin J, Scangas C et al (2013) Reducing antibiotic use in chinese pig farms. Gt Probl Semin Posters (All Posters, All Years), p 333. https://digit alcommons.wpi.edu/gps-posters/333

de Gara C, Burget DW, Sivakumaran T, Hunt RH (1986) The effect of temperature and $\mathrm{pH}$ on the stability of human pepsin in stored gastric juice: a method to prevent activity loss. Scand J Gastroenterol 21:650-654. https ://doi.org/10.3109/00365528609011096

Dong H, Dong Y, He L et al (2007) Studies on constituents and anti-inflammatory activity of rhizoma Atractylodis macrocephalae. Chinese Pharm J 42(14):1055-1059

Eyssen H (1973) Role of the gut microflora in metabolism of lipids and sterols. Proc Nutr Soc 32:59-63

Ferguson D, Smith T, Hanson B, Wardyn S, Donham KJ (2016) Detection of airborne methicillin-resistant Staphylococcus aureus inside and downwind of a swine building, and in animal feed: potential occupational, animal health, and environmental implications. J Agromed. https://doi. org/10.1080/1059924x.2016.1142917

Frick J, Autenrieth IB (2012) The gut microflora and its variety of roles in health and disease. Curr Top Microbiol Immunol. https://doi. org/10.1007/82_2012_217

Fu Y, Wang Y, Zhang B (2015) Systems pharmacology for traditional Chinese medicine with application to cardio-cerebrovascular diseases. J Tradit Chinese Med Sci. https://doi.org/10.1016/j.jtcms.2014.09.006

Fuchs SM, Heinemann C, Schliemann S, Härtl H, Fluhr J, Elsner P (2006) Assessment of anti-inflammatory activity of Poria cocos in sodium lauryl sulphate-induced irritant contact dermatitis. Skin Res Technol 12:223227. https://doi.org/10.1111/j.0909-752X.2006.00168.x

Funderburke DW, Seerley RW (1990) The effects of postweaning stressors on pig weight change, blood, liver and digestive tract characteristics. J Anim Sci 68:155-162. https://doi.org/10.2527/1990.681155x

Gao HX, Chen GS, Xu ZF (2010) Effects of compound Chinese herbal medicine on growth performance and blood biochemical index of weaning Xiangpig. Guizhou Agric Sci 38(8):146-148

Gellin G, Langlois BE, Dawson K, Aaron DK (1989) Antibiotic resistance of Gram-negative enteric bacteria from pigs in three herds with different histories of antibiotic exposure. Appl Environ Microbiol 55:2287-2292

Geng F, Wang W, Zhou T (2011) Antibacterial mechanisms of Fructus mume extract against Listeria innocua. Food Sci 32:88-93

Goldin B (1990) Intestinal microflora: metabolism of drugs and carcinogens. Ann Med 22:43-48. https://doi.org/10.3109/07853899009147240

Hamscher G, Theresia Pawelzick H, Sczesny S, Nau H, Hartung J (2003) Antibiotics in dust originating from a pig-fattening farm: a new source of health hazard for farmers? Environ Health Perspect 111:1590-1594. https://doi. org/10.1289/ehp.6288

Han X, Ji X, Zhao H, Zhang Y, Liu G, Wang Y, Zhao W, Wang S (2017) on the mechanism of coix seed compositions in treatment of spleen deficiency and wet dampness Zheng. Afr J Tradit Complement Altern Med 14:239-246. https://doi.org/10.21010/ajtcam.v14i4.26

He DY, Dai SM (2011) Anti-inflammatory and immunomodulatory effects of Paeonia lactiflora Pall., a traditional chinese herbal medicine. Front Pharmacol 2:10

Hsiao WLW, Liu L (2010) The Role of traditional chinese herbal medicines in cancer therapy - from tcm theory to mechanistic insights. Planta Med 76:1118-1131

Hui S Y, Weng B C, Tu F L (2011) Effects of Chinese traditional herbal medicine complex supplementation on the growth performance, immunity and serum traits of pigs. Anim Sci J 82(6):747-752

Hyun Y, Ellis M, Riskowski G, Johnson RW (1998) Growth performance of pigs subjected to multiple concurrent environmental stressors. J Anim Sci 76:721-727

Jin LM, Qing-Lin WU, Jin BF, Fang GY, Liu HB, Lin ZP, Wang MH, Gao-Min MA, Dai H (2008) Influence of anti-heat stress Chinese herbal medicine additive on amount of milk and blood biochemical parameters in dairy cow. Chinese J Vet Sci 28:852-857

Jin R, Ye F, Yu QP (2011) Studies on the main nutrient components of Coat. Jilin Agric 8:48

Kaneda T, Hidaka Y, Kashiwai T, Tada H, Takano T, Nishiyama S, Amino N, Miyai K (1992) Effect of coix seed on the changes in peripheral lymphocyte subsets. Rinsho Byori 40:179-181 
Lallès JP, Boudry G, Favier C, Le Floc'h N, Luron I, Montagne L, Oswald IP, Pié S, Piel C, Sève B (2002) Gut function and dysfunction in young pigs: physiology. Physiology 53:301-316

Lee HJ, Lee MH, Han IK (2000) Application of ELISA for the detection of penicillin antibiotic residues in live animal. Asian Aust J Anim Sci 13:1604-1608. https://doi.org/10.5713/ajas.2000.1604

Li L, Gong DQ, Zhang J, Chu DS, Liu ZH (2008) Effect of chinese herb medicine feed additive on immune performance of chinese yellow-feathered chicken. China Anim Husb Vet Med 35:26-28

Liu YQ, Liang J, Yang ZC, Liu BQ, Jin LM, Wz H (2010) Study progress on the pharmacological functions of Coix seed. J Anhui Agric Sci 38:10685-10686

Liu S, Li F, Zhang X (2019) Structural modulation of gut microbiota reveals Coix seed contributes to weight loss in mice. Appl Microbiol Biotechnol. https ://doi.org/10.1007/s00253-019-09786-z

Lucas IA, Calder AF, Smith H (1959) The early weaning of pigs IV. Comparisons of levels of antibiotic and sources of protein in diets for pigs weaned at 9 lb. live weight. J Agric Sci 53:125-129. https://doi.org/10.1017/S0021 859600030963

Luo Z, Huang CG, Sha T (2016) Report on finishing experiment of coix seed shell on beef cattle. Rur Sci and Tech 35:34-35

Moeser A, Pohl C, Rajput M (2017) Weaning stress and gastrointestinal barrier development: Implications for lifelong gut health in pigs. Anim Nutr. https://doi.org/10.1016/j.aninu.2017.06.003

Murphy M, Sanderson W, Vargo J (2007) Airborne antibiotic concentrations in a swine feeding operation. J Agric Saf Health 13:357-366. https://doi. org/10.13031/2013.23922

Normile D (2003) The new face of traditional chinese medicine. Science 299:188-190

Pettigrew J (2012) Health management with reduced use of antibiotics in pig production 9:130-138

Qu D, Sun W, Liu M, Liu Y, Zhou J, Chen Y (2016) Bitargeted microemulsions based on coix seed ingredients for enhanced hepatic tumor delivery and synergistic therapy. Int J Pharm. https://doi.org/10.1016/j.jpha rm.2016.03.001

Ramos C, Thorsen L, Ryssel M, Nielsen D, Siegumfeldt H, Schwan R, Jespersen $L$ (2014) Effect of the gastrointestinal environment on $\mathrm{pH}$ homeostasis of Lactobacillus plantarum and L. brevis cells as measured by real-time fluorescence ratio-imaging microscopy. Res Microbiol. https://doi. org/10.1016/j.resmic.2014.02.005

Sapkota A, Ojo K, Roberts M, Schwab KJ (2006) Antibiotic resistance genes in multidrug-resistant Enterococcus spp. and Streptococcus spp. recovered from the indoor air of a large-scale swine-feeding operation. Lett Appl Microbiol 43:534-540. https://doi.org/10.1111/j.1472-765X.2006.01996.X

Spiro DM, Welker MA, Arnold DH, Meckler GD (2011) A proposal to limit otoscopy to reduce unnecessary use of antibiotics: a call for research. Expert Rev Anti Infect Ther 9:177-181

Stahly TS, Cromwell GL, Monegue HJ (1981) Effects of the dietary inclusion of copper and(or) antibiotics on the performance of weanling pigs. J Anim Sci 51:1347-1351. https://doi.org/10.2527/jas1981.5161347x

Stein HH, Dong YK (2006) Reduced use of antibiotic growth promoters in diets fed to weanling pigs: dietary tools, part 2. Anim Biotechnol 17:217-231

Thexton AJ, Crompton A, German R (1998) Transition from suckling to drinking at weaning: a kinematic and electromyographic study in miniature pigs. J Exp Zool 280:327-343. https://doi.org/10.1002/(SICI)1097-010X(19980 401)280:5\%3c327:AID-JEZ2\%3e3.0.CO;2-Q

Tokuda H, Matsumoto T, Konoshima T, Kozuka M, Nishino H, Iwashima A (1990) Inhibitory effects on Epstein-Barr virus activation and anti-tumor promoting activities of coix seed. Planta Med 56:653-654
Van Der Fels-Klerx HJ, Puister-Jansen LF, Van Asselt ED, Burgers SLGE (2011) Farm factors associated with the use of antibiotics in pig production. J Anim Sci 89:1922-1929

Velagapudi VR, Hezaveh R, Reigstad CS, Gopalacharyulu P, Yetukuri L, Islam S, Felin J, Perkins R, Borã@N J, Oresic M (2010) The gut microbiota modulates host energy and lipid metabolism in mice. J Lipid Res 51:1101-1112

Vente-Spreeuwenberg MAM, Beynen AC (2003) Diet-mediated modulation of small intestinal integrity in weaned piglets, Weaning the pig concepts \& consequences, vol 7, pp 145-198

Wang Q, Du Z, Zhang H, Zhao L, Sun J, Zheng X, Ren F (2015) Modulation of gut microbiota by polyphenols from adlay (Coix lacryma-jobi L. var. mayuen Stapf.) in rats fed a high-cholesterol diet. Int J Food Sci Nutr 66:1-7. https://doi.org/10.3109/09637486.2015.1088941

Wang TT, Zhong LY, Ting XU, Pharmacy SO (2017) The influence on the antidiarrheal effect of mice and gastrointestinal motility by processing Coptidis Rhizome with fresh ginger juice, dry ginger juice and different ginger juice. Lishizhen Med Mater Medica Res 08:12-19

Weng CJ (2013) Effects of coix on growth performance of rabbits and pigs. Feed Expo 10:37-39

Williamson E, Lorenc A, Booker A, Robinson N (2013) The rise of Traditional Chinese Medicine and its materia medica: a comparison of frequency and safety of materials and species used in Europe and China. J Ethnopharmacol. https://doi.org/10.1016/j.jep.2013.06.050

Wolter BF, Ellis M, Corrigan BP, Dedecker J (2002) The effect of birth weight and feeding of supplemental milk replacer to piglets during lactation on preweaning and postweaning growth performance and carcass characteristics. J Anim Sci 80:301-308. https://doi.org/10.2527/2002.802301x

Yang ZC, Liang J, Liu YQ (2011) Analysis of the constituents of Areaea chinensis. Anhui Agri Sci 39(2):756

Yang H, Xia X, Yin Y (2013) Development and renewal of intestinal villi in pigs. In: Nutritional and physiological functions of amino acids in pigs. Part l. pp. 29-39

Yu Y-L, Lu Y, Tang X, Cui F-D (2008) Formulation, preparation and evaluation of an intravenous emulsion containing brucea javanica oil and coix seed oil for anti-tumor application. Biol Pharm Bull 31:673-680. https://doi. org/10.1248/bpb.31.673

Zhang L-P (2005) Exploration on the benefit and function of Pericarpium citri reticulatae. Zhongguo Zhong Xi Yi Jie He Za Zhi 25:754-757

Zhang J, Zhang X, Liang X, Gu H, Zhu P (2008) Effects of different Chinese herbal medicines on biochemical parameters in guinea-pig with pigment gallstones. Zhong Xi Yi Jie He Xue Bao 6:856-859. https://doi. org/10.3736/jcim20080816

Zhao L, Zhao A-G, Zhao G, Xu Y, Zhu X-H, Cao N-D, Zheng J, Yang J-K, Xu J-H (2014) Survival benefit of traditional chinese herbal medicine (a herbal formula for invigorating spleen) in gastric cancer patients with peritoneal metastasis. Evid Based Complement Altern Med. https://doi. org/10.1155/2014/625493

Zhou Z, Yang J, Kong A-N (2017) Phytochemicals in traditional chinese herbal medicine: cancer prevention and epigenetics mechanisms. Curr Pharmacol Reports. https://doi.org/10.1007/s40495-017-0086-1

\section{Publisher's Note}

Springer Nature remains neutral with regard to jurisdictional claims in published maps and institutional affiliations. 